\title{
The ways to increase reliability of work of powerful electrical machines in autonomous electric networks
}

\author{
Ilya Boguslawsky ${ }^{1, *}$ and Valery Zaboin ${ }^{1}$ \\ ${ }^{1}$ Peter the Great Saint Petersburg Polytechnic University, Polytechnicheskaya str. 29, Saint \\ Petersburg, 195251, Russia
}

\begin{abstract}
The influence of the harmonic composition of the stator current on the permissible power of an electric machine during its operation in an autonomous nonlinear network is considered. A generalizing equation is proposed for calculating this power, taking into account an increase in the electric losses in the stator winding and losses in the steel of the machine. The analysis of the causes of voltage drop at the terminals of an autonomous generator is made and practical recommendations for its reduction are given for an unexpected change in the load of the generator.

The main purpose of investigation is to obtain a generalized expression is derived for calculating the permissible that makes to estimate a reduction of the nominal power of power of alternating current machine when operating in nonlinear networks in condition of knowing harmonics of the stator current. It is usefully for newly created generators and can suppose a changing the operational requirements by the standards for the to the value of transient deviation of the voltage when the load changes suddenly.
\end{abstract}

\section{Introduction}

A characteristic feature of the electric machines operation in autonomous electrical networks with nonlinear semiconductor elements (rectifiers, inverters, static frequency converters) is an increase of the stator current higher harmonics in comparison with operation in large power linear networks, which leads to an increase in the additional losses in the construction elements and the appearance of additional alternating moments on the shaft, as a result of which are the windings and active steel heating and the increasing the magnitudes of vibrations [1-4]. Among the problems that arise in ensuring the operational reliability of machines are the tasks of determining the permissible values of their power in various operating modes, as well as evaluating the possible level of voltage drop in generators of autonomous systems during sudden changes in the load in condition working with nonlinear load. The paper presents the results of solving these problems [5-10]. In this article we will obtain some expressions allow us to estimate the permissible a reduction of the nominal

\footnotetext{
*Corresponding author: $\underline{\text { b-iz@yandex.ru }}$
} 
power of power of alternating current machine when operating in nonlinear networks in condition of knowing harmonics of the stator current.

\section{Materials and Methods}

\subsection{Estimating the permissible power of an alternating current machine in autonomous non-linear electrical networks}

The relative value of the permissible power of the machine $P_{\text {per }}$, when it operates in autonomous nonlinear networks, can be represented as the product of two factors $\underline{P}_{\mathrm{pc}}$ and $\underline{P}_{\mathrm{ps}}$, the first of which takes into account the reduction in the rated power of the machine due to the increasing losses in the stator winding (losses in copper), and the second for increasing losses in the active steel of the stator and the rotor. From the condition of equality of losses (for preserving the permissible values of the machine elements overheating when it operates in linear and nonlinear networks), it is possible, by transforming the equations obtained earlier in [2], to represent the expression for calculating $\underline{P}_{\mathrm{pc}}$ in the form

$$
\underline{P}_{p c}=\sqrt{\frac{k_{F 1}}{\sum \boldsymbol{k}_{v}^{2}+\left(\boldsymbol{k}_{F 1}-1\right) \sum \boldsymbol{k}_{v}^{2} \cdot v^{2}}},
$$

where $k_{F 1}$ is the Field coefficient, and $k_{v}$ is the ratio of the amplitude of the $v$-th harmonic current to the amplitude of its first harmonic current.

Performing similar transformations with the corresponding equations in [2], the expression for determining $\underline{P}_{\mathrm{ps}}$ can be written as

$$
\underline{P}_{p s}=\frac{1}{\sqrt{\sum k_{s v}^{2} \cdot k_{v}^{2} \cdot v^{1.3}}}
$$

where $k_{s v}=s_{v} / s_{1}$, and $s_{v}$ is the ratio of the amplitudes of the $v$-th magnetomotive force harmonics of the resultant magnetic field $F_{\delta v}$ and the field of the stator winding $F_{a v}$ to the saturation coefficient of the magnetic circuit $k_{\mu}[2]$.

Taking into account (1) and (2), the generalizing formula for calculating the permissible power of the machine $\underline{P}_{\text {per }}$ takes the form

$$
\underline{P}_{p e r}=\sqrt{\frac{k_{F 1}}{\left(\sum \boldsymbol{k}_{s v}^{2} \cdot \boldsymbol{k}_{v}^{2} \cdot v^{1 \cdot 3}\right) \cdot\left(\sum \boldsymbol{k}_{v}^{2}+\left(k_{F 1}-1\right) \cdot \sum \boldsymbol{k}_{v}^{2} \cdot v^{2}\right)}} .
$$

It is important to emphasize that in synchronous machines the value of $s_{1}$ in (2) and (3) is inversely proportional to the inductive reactance of the armature reaction $x_{a d}$, and in asynchronous and synchronous motors with a complete damper system it is less than 0.1 [3]. However, for $s_{v}<1$, the corresponding (first) sum in the denominator (3) and the value of $\underline{P}_{\mathrm{ps}}$ in (2) tend to 1, and therefore, in engineering calculations it is allowed to take into account the reduction in the machine power only due to the increasing losses in the stator winding using (1), or consider $\underline{P}_{\mathrm{ps}}$ equal to $0.95-0.9$ for current distortion coefficients $k_{i}>1.2$.

Analysis (3) and operational experience have shown that for the increase $\underline{P}_{\mathrm{per}}$ in the range up to $0.93-0.95,12$-pulse frequency converters should be used to supply large power motors, and a six-phase stator winding should be used in the generators.

\subsection{Estimation of the transient voltage deviation of an autonomous generator with a sudden change in loading}

The most important requirement for generators operation in autonomous power supply systems is the permissible value of its transient deviation of the voltage $\Delta U$ when the load changes suddenly. Using the formulas $[9,10]$ and results of processing experimental data for 
a number of large power generators, we can obtain an approximate expression of the transient voltage deviation $\Delta U$ for a $100 \%$ load change with $\cos \varphi \leq 0.4$ in the form

$$
\Delta U \approx x^{*} /\left(1+x^{*}\right)
$$

where $x^{*}$ is the value of the calculated inductive resistance, lying in the range from the value of the supertransient inductive resistance $x_{d}^{\prime \prime}$ to the value of the transient inductive resistance $x_{d}^{\prime}$ along the longitudinal axis. And from (4) it follows that $\Delta U$ is less than admissible $15 \%$ is reached at a value of $x^{*} \leq 0.18$. Therefore the task of decreasing $\Delta U$ resolves into a decrease of the resistances along the paths of the longitudinal magnetic flux scattering of the stator winding since it is known [11] that

$$
x_{d}^{\prime \prime}=x_{\sigma a}+\frac{1}{\frac{1}{x_{a d}}+\frac{1}{x_{\sigma f}}+\frac{1}{x_{\sigma y d}}},
$$

where $x_{a d}$ is the inductive resistance of the armature longitudinal response, and $x_{\sigma a}, x_{\sigma f}$ and $x_{\sigma y d}$ are the inductive resistances of the armature, excitation and damping winding.

Each of the resistances in (5) can be represented as $k \cdot \frac{A}{B_{\delta 1}}$, where $k$ takes into account the geometry and saturation of the scattering paths of the corresponding magnetic fluxes, $A$ is the linear current load of the stator winding, and $B_{\delta 1}$ is the induction first harmonic amplitude of the magnetic field in the gap [11-13]. Therefore, in order to reduce $\Delta U$, it is necessary to reduce either magnetic conductivities for winding fluxes, or the ratio of the main electromagnetic loads $A$ and $B$.

The practice of calculating modern generators for autonomous power supply systems shows [14-25] the first method allows reducing $\Delta U$ by no more than $5-6 \%$. If the generator voltage drop exceeds the permissible level $\Delta U$, then the second method can also be used, since the increase of $B_{\delta 1}$ by a factor of $n$ with a constant power in the specified generator dimensions and the corresponding decrease of $A$ by a factor of $n$ (to maintain the force unchanged $\sigma_{t}=\alpha \cdot A B \delta_{1}$ ) reduces $x^{*}$ by $n^{2}$ times. However, the calculations showed that this method allows to reduce $\Delta U$ by only $10-12 \%$, and a further increasing of $n$ will lead to unacceptable overheating of the windings, deterioration of electromagnetic use, and performance of the generator. Moreover, the decrease in $A$ just contradicts the tendency for the modern electric machine industry to increase the level of electromagnetic use of generators by increasing $A$ in order to reduce their mass $G_{g}$ and the dimensions of $V_{g}$, which leads to an increase in $\Delta U$ exceeding the operational requirements of existing standards for autonomous systems power supply.

For newly created generators with $x_{d}^{\prime}>0.18$, the solution of this problem is possible only by reducing the current level of the inductive resistances in accordance with the required value of the voltage drop $\Delta U$.

For generators in operation, this task should be solved by separating two or three subgroups of consumers that differ by their technological necessity. As a result, the required $\Delta U_{g r}<15 \%$ can be reached with a high level of electromagnetic use of each generator in the group $\left(x_{d}^{\prime} \approx 0.35-0.4\right.$ ), since the equivalent inductive resistances of the group are proportional to the ratio of the connected power $P_{2 g r}$ to the power $P_{1 n}$ of the generator, that is $x_{g r}^{*} x^{*} \cdot P_{2 g r} / P_{1 n}$ and, as a consequence of (4) $\Delta U_{g r}<\Delta U$.

\section{Results}

1. A generalized expression is derived for calculating the permissible power of an alternating current machine $\bar{P}_{p e r}$, which makes it possible to estimate the necessary reduction in its nominal power when operating in nonlinear networks with a known harmonics of the stator current. 
2. The tendency to increase the level of electromagnetic use of generators contradicts to the operational requirements of the current standards for the permissible value of its transient deviation of the voltage $\Delta U$ with a sudden load change.

3. For newly created autonomous system generators, it is necessary to change the operational requirements of the standards to the value of $\Delta U$ or to the value of the projected load $P_{2}$, taking into account the levels of electromagnetic loads and parameters reached in the generators.

4. The required value of $\Delta U$ in existing autonomous networks can be reached by dividing the load into subgroups with $P_{2 \mathrm{gr}}$, taking into account the proportionality of the equivalent inductive resistances to the ratio $P_{2 g r} / P_{1 \text { nom }}$.

\section{References}

1. I. Boguslawsky, Proceedings of RAS. Power Engineering 2, 21-26 (2009)

2. I. Boguslawsky, Electrical Engineering 5, 22-28 (2009)

3. I. Arseniev, I. Boguslawsky, V. Popov, V. Sukhanov, Scientific and Technical sheets of the Saint Petersburg State Polytechnic University 4-1, 41-48 (2013)

4. I. Arseniev, I. Boguslawsky, N. Korovkin, Proceedings of RAS. Power Engineering, 4, 22-35 (2013)

5. I. Boguslawsky, V. Zaboin, V. Popov, Scholarly notes Komsomolsk-na-Amure State University 4 (20), 12-16 (2014)

6. G. Chesnokov, V. Zaboin, Scientific and Technical sheets of the Saint Petersburg State Polytechnic University 2, 72-79 (2011)

7. I. Boguslawsky, N. Korovkin, M. Hayakawa, Large A.C. Machines: Theory and investigation methods of currents and losses in stator and rotor meshes (Springer, New York, 2016)

8. I. Boguslawsky, N. Korovkin, I. Kruchinina, V. Paltseva, Bulletin of the Pskov State University. Series: Engineering 4, 33-50 (2016)

9. A. Vazhnov, Transients in AC machines (Energia, Leningrad,1980)

10. R. Rudenberg, Operational Regimes of Electric Power Systems and Installations (Energia, Leningrad, 1981)

11. A. Woldek, V. Popov, Electric machines. Alternating current machines. (Piter, St. Petersburg, 2008)

12. I. Boguslavsky, N. Korovkin, Proceedings of RAS. Power Engineering 2, 57-68 (2015)

13. I. Boguslavsky, N. Korovkin, Proceedings of RAS. Power Engineering, 4, 12-21 (2013)

14. N. Korovkin, S. Krivosheev, S. Shishigin, T. Minevich, K. Netreba, Proceedings of RAS. Power Engineering 6, 80-89 (2013)

15. A. Adalev, N. Korovkin, M. Hayakawa, IEEE Transactions on Microwave Theory and Techniques 54(7), 3131-3139 (2006)

16. N. Korovkin, J. Nitsch, H.-J. Scheibe, IEEE International Symposium on EMC 2, 1429120, 1148-1151 (2003)

17. N. Korovkin, A. Adalev, M. Hayakawa, IEEE Trans. on Circuits and Systems I: Fundamental Theory and Applications 55(5), 1237-1247 (2008)

18. S. Dubitsky, G. Greshnyakov, N. Korovkin, IEEE International Energy Confernce, ENERGYCON 2016, 14 July 2016, № 7514107(2016). 
19. S. Dubitsky, G. Greshnyakov, N. Korovkin, 9th Int. Electric Power Quality and Supply Reliability Conference, 6866837, 341-346 (2014)

20. N. Korovkin, O. Frolov, E. Ivanova, S. Shishiguin, IEEE International Symposium on Electromagnetic Compatibility, 6396752 (2012)

21. N. Belyaev, N. Korovkin, O. Frolov, V. Chudnyi, Russian Electrical Engineering 2, 2128 (2013)

22. N. Belyaev, A. Egorov, N. Korovkin, V. Chudnyi, Proceedings of the Scientific and Technical Center of the Russian Unified Energy System 2(69), 50-67 (2013)

23. N. Belyaev, N. Korovkin, V. Chudnyi, Elektrichestvo. 2, 18-24 (2014)

24. N. Korovkin, O. Frolov, Proceedings of the Russian Academy of Sciences. Power industry 6, 39-47 (2013)

25. N. Korovkin, S. Gritsutenko, Proceedings of the Russian Academy of Sciences. Power Engineering 2, 73-86 (2017) 